\title{
Moderate hypothermia during aortic arch surgery is associated with reduced risk of early mortality
}

\author{
January Y. Tsai, MD, ${ }^{\text {a }}$ Wei Pan, MD, ${ }^{\text {a,c }}$ Scott A. LeMaire, MD, ${ }^{\text {b,d }}$ Paul Pisklak, MD, ${ }^{\text {a }}$ Vei-Vei Lee, MS, ${ }^{\mathrm{e}}$ \\ Arthur W. Bracey, MD, ${ }^{\mathrm{f}}$ MacArthur A. Elayda, MD, PhD, ${ }^{\mathrm{e}}$ Ourania Preventza, MD, ${ }^{\mathrm{b}, \mathrm{d}}$ Matt D. Price, MS, ${ }^{\mathrm{b}, \mathrm{d}}$ \\ Charles D. Collard, MD, ${ }^{\mathrm{a}, \mathrm{c}}$ and Joseph S. Coselli, $\mathrm{MD}^{\mathrm{b}, \mathrm{d}}$
}

\begin{abstract}
Objective: Selective antegrade cerebral perfusion (ACP) during hypothermic circulatory arrest (HCA) provides cerebral protection during aortic arch surgery. However, the ideal temperature for HCA during ACP remains unknown. Clinical outcomes were compared in patients who underwent moderate (nasopharyngeal temperature, $\geq 20^{\circ} \mathrm{C}$ ) versus deep (nasopharyngeal temperature, $<20^{\circ} \mathrm{C}$ ) $\mathrm{HCA}$ with ACP during aortic arch repair.
\end{abstract}

\begin{abstract}
Methods: By using a prospectively maintained clinical database, we analyzed data from 221 consecutive patients who underwent aortic arch replacement with HCA and ACP between December 2006 and May 2009. Seventy-eight patients underwent deep hypothermia (mean lowest temperature, $16.8^{\circ} \mathrm{C} \pm 1.7^{\circ} \mathrm{C}$ ) and 143 patients underwent moderate hypothermia (mean, $22.9^{\circ} \mathrm{C} \pm 1.4^{\circ} \mathrm{C}$ ) before systemic circulatory arrest was initiated. Multivariate stepwise logistic and linear regressions were performed to determine whether depth of hypothermia independently predicted postoperative outcomes and blood-product use.
\end{abstract}

Results: Compared with moderate hypothermia, deep hypothermia was associated independently with a greater risk of in-hospital death $(7.7 \%$ vs $0.7 \%$; odds ratio [OR], 9.3; 95\% confidence interval [CI], 1.1-81.6; $P=.005)$ and 30 -day all-cause mortality $(9.0 \%$ vs $2.1 \%$; OR, $4.7 ; 95 \%$ CI, $1.2-18.6 ; P=.02)$, and with longer cardiopulmonary bypass time (154 \pm 62 vs $140 \pm 46 \mathrm{~min} ; P=.008)$. Deep hypothermia also was associated with a higher incidence of stroke, although this association was not statistically significant $(7.6 \%$ vs $2.8 \%$; $P=.073 ; \mathrm{OR}, 4.3 ; 95 \% \mathrm{CI}, 0.9-12.5)$. No difference was seen in acute kidney injury, blood product transfusion, or need for surgical re-exploration.

Conclusions: Moderate hypothermia with ACP is associated with lower in-hospital and 30-day mortality, shorter cardiopulmonary bypass time, and fewer neurologic sequelae than deep hypothermia in patients who undergo aortic arch surgery with ACP. (J Thorac Cardiovasc Surg 2013;146:662-7)

Hypothermic circulatory arrest (HCA) has been used for decades for cerebral protection during aortic arch surgery. HCA creates a motionless, bloodless field; minimizes the need for aortic clamping; and provides neuroprotection by reducing the cerebral metabolic rate, excitatory transmitter release, ion influx, and vascular permeability. ${ }^{1}$ However, the ideal temperature for HCA remains unknown. Prolonged deep HCA (DHCA) has been associated with increased coagulopathy, inflammatory response, vascular endothelial dysfunction, apoptosis, and stroke risk. ${ }^{2-4}$ Interestingly,

\footnotetext{
From the Departments of Anesthesiology ${ }^{\mathrm{a}}$ and Surgery, ${ }^{\mathrm{b}}$ Baylor College of Medicine; the Divisions of Cardiovascular Anesthesiology ${ }^{\mathrm{c}}$ Cardiovascular Surgery, ${ }^{\mathrm{d}}$ Biostatistics and Epidemiology, ${ }^{\mathrm{e}}$ and Pathology, ${ }^{\mathrm{f}}$ the Texas Heart Institute at St. Luke's Episcopal Hospital, Houston, Tex.

Support was provided solely by institutional and departmental sources.

Disclosures: Authors have nothing to disclose with regard to commercial support.

Received for publication Aug 30, 2012; revisions received Feb 18, 2013; accepted for publication March 5, 2013; available ahead of print April 3, 2013.

Address for reprints: Wei Pan, MD, Department of Cardiovascular Anesthesiology, the Texas Heart Institute at St. Luke's Episcopal Hospital, PO Box 20345, Houston, TX 77030 (E-mail: wpan@texasheart.org).

0022-5223/\$36.00

Copyright (c) 2013 by The American Association for Thoracic Surgery

http://dx.doi.org/10.1016/j.jtcvs.2013.03.004
}

studies have suggested that deep hypothermia alone without circulatory arrest also may be associated with increased inflammatory response and neurologic damage. ${ }^{5-7}$

With the advent of antegrade cerebral perfusion (ACP) during HCA to provide some measure of uninterrupted cerebral perfusion during aortic arch repair, the desired temperature level for HCA has become even more unclear. Indeed, moderate HCA (MHCA) (nasopharyngeal temperature, $\geq 20^{\circ} \mathrm{C}$ ) still may afford cerebral protection while minimizing the side effects of DHCA (nasopharyngeal temperature, $<20^{\circ} \mathrm{C}$ ). To address this question, we compared clinical outcomes in patients who underwent MHCA versus DHCA with selective ACP during aortic arch repair.

\footnotetext{
MATERIALS AND METHODS Study Design

Institutional review board approval and informed patient consent were obtained for the prospective collection and subsequent analysis of clinical data. We identified 243 consecutive patients who underwent aortic arch repair between December 2006 and May 2009 at the Texas Heart Institute at St. Luke's Episcopal Hospital (Houston, Tex). Both HCA and selective ACP were used in all patients. Patients who received aprotinin intraoperatively $(\mathrm{n}=22)$ were excluded from further analysis because of this drug's
} 


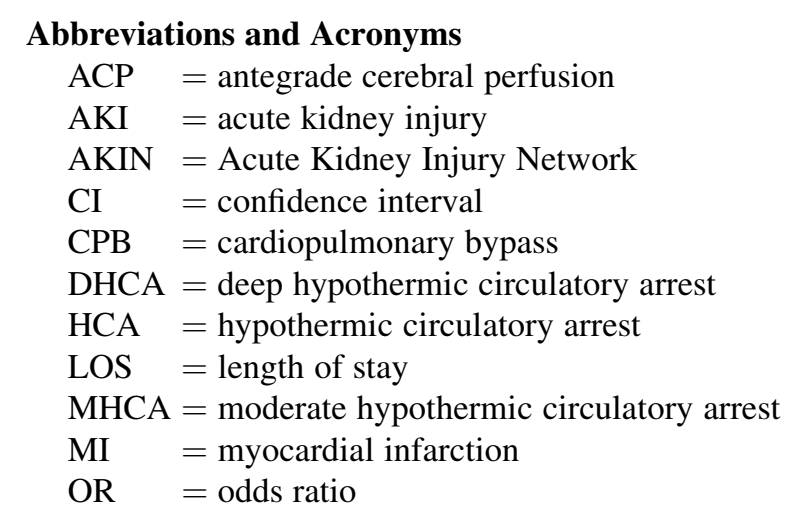

adverse effects on renal function and mortality risk. ${ }^{8}$ The 221 remaining patients were divided into 2 groups: DHCA (lowest nasopharyngeal temperature, $<20^{\circ} \mathrm{C} ; \mathrm{n}=78$ ) and MHCA (lowest nasopharyngeal temperature, $\geq 20^{\circ} \mathrm{C} ; \mathrm{n}=143$ ).

\section{Data Collection}

Patient demographics, perioperative risk factors, and the incidence of adverse clinical outcomes were abstracted from a prospectively maintained clinical database. Preoperative renal function was assessed by using the abbreviated Modification of Diet in Renal Disease study equation to calculate each patient's estimated glomerular filtration rate. ${ }^{9}$ Patient temperature was defined as the lowest recorded intraoperative nasopharyngeal temperature, which generally coincided with HCA initiation. Measured adverse outcomes included in-hospital mortality, 30-day all-cause mortality, stroke, myocardial infarction (MI), acute kidney injury (AKI), and postoperative bleeding that necessitated reoperation. A diagnosis of MI was made if the records showed new Q waves (Minnesota code, 1-1-1 to 1-2-7), new persistent ST-segment or T-wave changes (Minnesota code, 4-1, 4-2, 5-1, 5-2, or 9-2), increased levels of the muscle-brain isoenzyme of creatine kinase, or evidence of acute MI on autopsy. Stroke was diagnosed if there was clinical evidence of it or if a new focal or global defect was observed on computed tomography, magnetic resonance imaging, or autopsy. Postoperative AKI was defined according to Risk, Injury, Failure, Loss, and End-stage kidney diseases criteria ${ }^{10}$ and Acute Kidney Injury Network (AKIN) classification. ${ }^{11}$ Survivors' hospital length of stay (LOS) was computed as the number of days between procedure and discharge, including any time patients spent at hospitals or long-term acute care facilities after transfer from our institution. The number of units of packed red blood cells, fresh-frozen plasma, pooled platelets, platelet apheresis, and cryoprecipitate administered within 48 hours of surgery was collected from blood bank records.

\section{Surgical and Anesthetic Technique}

All patients underwent aortic arch replacement to treat aortic arch aneurysm or dissection. Before general anesthesia was induced, 2-channel near-infrared spectroscopy (INVOS System; Somanetics, Troy, Mich) noninvasive probes were placed on the forehead, approximating the frontal lobes bilaterally. The near-infrared spectroscopy then was monitored throughout surgery. General anesthesia was performed in a standardized fashion with etomidate, midazolam, fentanyl, and isoflurane. Before incision, patients received either aminocaproic acid or tranexamic acid as an antifibrinolytic.

All surgeries involved the use of HCA with selective ACP. Ninety-four percent of patients had either axillary artery cannulation or direct innominate artery cannulation to establish cardiopulmonary bypass (CPB). This was the case in $95 \%$ of elective procedures and $91 \%$ of urgent or emergent procedures. After systemic heparinization (300 IU/kg), CPB and systemic cooling were initiated. For $\mathrm{CPB}, \mathrm{pH}$-stat principles of acid-base management were used. Repeated administration of cold cardioplegia solution and topical cooling were used for myocardial protection. During cooling, $100 \mathrm{mg}$ lidocaine, $100 \mathrm{mg}$ esmolol, $150 \mathrm{mg}$ amiodarone, and $2 \mathrm{mg}$ magnesium were administered routinely through the central line. In the DHCA group, cooling continued until electrocerebral silence was seen on multichannel electroencephalography (mean nasopharyngeal temperature, $16.8^{\circ} \mathrm{C} \pm 1.7^{\circ} \mathrm{C}$ ). Starting in January 2008 , the target temperature for HCA was increased to $23^{\circ} \mathrm{C}$; all patients treated after this change was made were included in the MHCA group.

In patients undergoing total aortic arch repair, the Y-graft technique (ie, arch replacement with bifurcated or trifurcated grafts) was used. ${ }^{12}$ Briefly, 2 of the branches of the Y-graft were sutured to the left subclavian artery and the left common carotid artery during the cooling phase. Upon reaching the target temperature, $500 \mathrm{mg}$ of thiopental was administered, the patient's head was packed in ice, and HCA was initiated with ACP (target flow, $10-20 \mathrm{~mL} / \mathrm{kg} / \mathrm{min}$ ) via the right axillary artery (by clamping the innominate artery) and, sometimes, 1 or more branches of the Y-graft (Figure 1). The innominate artery then was divided and sewn to the distal end of the Y-graft. After the branch artery anastomoses were completed, the innominate artery clamp was removed and a clamp was placed on the proximal aspect of the Y-graft, restoring flow from the axillary artery to the attached brachiocephalic arteries. Graft replacement of the ascending aorta and transverse aortic arch then was performed.

After the distal aortic anastomosis was completed, CPB flow to the lower body was restarted through the axillary artery (in partial arch replacement cases) or a side branch of the arch graft (in total arch replacement cases). After the repair was completed, the patient was warmed to $36.5^{\circ} \mathrm{C}$ (avoiding blood-bath gradients of $>10^{\circ} \mathrm{C}$ ) and weaned from $\mathrm{CPB}$. Perioperative blood products were transfused according to the attending physician's judgment, taking into account the patient's clinical status and available laboratory values.

\section{Statistical Analysis}

All statistical analyses were performed with SAS statistical software (version 9.1; SAS Institute, Cary, NC) in the Division of Biostatistics and Epidemiology at the Texas Heart Institute (Houston, Tex). Continuous variables were expressed as the mean \pm standard deviation, and categoric (frequency) variables were expressed as percentages. Univariate logistic regression analyses initially were conducted to test for between-group differences in patient preoperative demographics, risk factors, and preoperative medications (Table 1). Categoric variables were analyzed with the $\chi^{2}$ or Fisher exact tests. Continuous variables first were examined for normality of distribution: the $t$ test was used for those variables with normal distributions, and the nonparametric Wilcoxon rank-sum test was applied for the variables with skewed distributions. In all tests, 2-tailed $P$ values were calculated.

To control for demographic factors, medications, and perioperative risk factors, multivariate stepwise logistic regression was performed on all patient data to determine whether DHCA was associated independently with worse postoperative outcomes than MHCA. Linear regression was used to determine whether DHCA was associated independently with prolonged CPB time, LOS, and increased blood product use.

\section{RESULTS}

Patient demographics and perioperative risk factors are presented in Table 1. Patients in the MHCA group had a marginally higher preoperative prevalence of pulmonary disease $(P=.07)$ and were more likely to undergo total arch repair $(P=.07)$ than patients in the DHCA group. Of note, the groups did not significantly differ with respect to age, gender distribution, diabetes, history of smoking, renal insufficiency, 


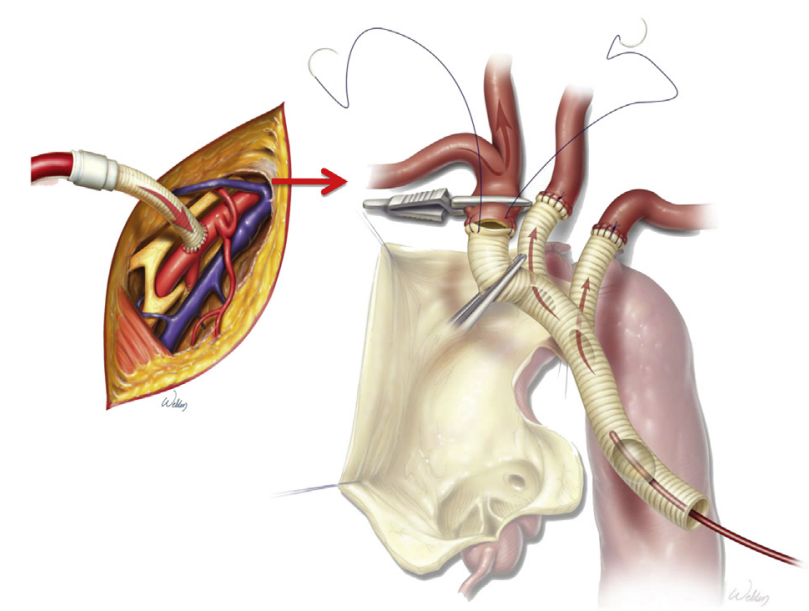

FIGURE 1. Cerebral perfusion approach used during hypothermic circulatory arrest.

hypertension, coronary artery disease, congestive heart failure, New York Heart Association class III/IV, history of stroke, emergent or urgent surgery, prior sternotomy, prior aortic surgery, or preoperative medications. Although HCA duration was not significantly different between groups, the

TABLE 1. Demographic variables and risk factors in patients ( $N=221$ ) undergoing aortic arch repair with DHCA or MHCA with antegrade cerebral perfusion

\begin{tabular}{lccc}
\hline $\begin{array}{c}\text { Demographic variables and risk } \\
\text { factors }\end{array}$ & $\begin{array}{c}\text { DHCA } \\
(\mathbf{n}=\mathbf{7 8})\end{array}$ & $\begin{array}{c}\text { MHCA } \\
(\mathbf{n}=\mathbf{1 4 3})\end{array}$ & $\boldsymbol{P}$ \\
\hline Age, y & $61 \pm 14$ & $60 \pm 15$ & .42 \\
Female sex, \% & 38 & 32 & .35 \\
Diabetes mellitus, \% & 9 & 6 & .34 \\
Preop serum creatinine level, mg/dL & $1.25 \pm 1.03$ & $1.26 \pm 0.98$ & .95 \\
Renal insufficiency, \% & 14 & 13 & .87 \\
Smoking history, \% & 33 & 41 & .29 \\
Hypertension, \% & 87 & 87 & .92 \\
Carotid disease, \% & 15 & 12 & .46 \\
Pulmonary disease, \% & 26 & 38 & .07 \\
Coronary artery disease, \% & 30 & 37 & .29 \\
Congestive heart failure, \% & 15 & 18 & .60 \\
NYHA class III/IV, \% & 33 & 36 & .70 \\
History of stroke, \% & 8 & 12 & .33 \\
Hemiarch or partial arch surgery, \% & 76 & 64 & .07 \\
Total arch surgery, \% & 24 & 36 & .07 \\
Emergent surgery, \% & 14 & 13 & .87 \\
Urgent surgery, \% & 12 & 12 & .94 \\
Prior sternotomy, \% & 28 & 36 & .26 \\
Acute aortic dissection, \% & 14 & 13 & .87 \\
First open aortic surgery, \% & 65 & 59 & .33 \\
CPB duration, min & $154 \pm 62$ & $140 \pm 46$ & .008 \\
HCA duration, min & $37 \pm 23$ & $38 \pm 25$ & .68 \\
B-Blocker, \% & 67 & 70 & .62 \\
Statins, \% & 37 & 40 & .70 \\
\hline NYHA, New York $\%$ & & 13 \\
\hline
\end{tabular}

NYHA, New York Heart Association; DHCA, deep hypothermic circulatory arrest; $M H C A$, moderate hypothermic circulatory arrest; $C P B$, cardiopulmonary bypass; $H C A$, hypothermic circulatory arrest.
DHCA group had significantly longer CPB times than the MHCA group ( $154 \pm 62$ vs $140 \pm 47 \mathrm{~min} ; P=.008)$.

Postoperative outcomes are summarized in Table 2. There were 7 in-hospital deaths (6 in the DHCA group and 1 in the MHCA group). Multiorgan system failure was the most common cause of death. There were 3 additional 30-day all-cause deaths (1 in the DHCA group and 2 in the MHCA group). To determine whether DHCA was associated independently with a significantly higher risk of in-hospital death and 30-day all-cause mortality, multivariate stepwise logistic analysis was performed, controlling for patient demographics, preoperative risk factors, and intraoperative variables. Deep HCA was associated independently with higher risks of in-hospital death ( $8 \%$ vs $1 \% ; P=.005)$ and 30-day all-cause mortality $(9 \%$ vs $2 \% ; P=.02$ ) than MHCA.

The incidence of stroke in the DHCA group was 3-fold higher than in the MHCA group $(8 \%$ vs $3 \% ; P=.07$; odds ratio $[\mathrm{OR}], 3.4 ; 95 \%$ confidence interval [CI], 0.912.6). However, the difference between groups did not reach statistical significance (probably because of the small sample size). There were 3 transient and 3 permanent strokes in the DHCA group, compared with 2 transient and 2 permanent strokes in the MHCA group. With respect to spinal cord injury, no paraplegia was observed in either group. Likewise, no significant differences between groups were observed with respect to the incidence of postoperative MI, atrial fibrillation, respiratory failure, need for postoperative tracheotomy, and postoperative bleeding necessitating reoperation (Table 2).

In terms of renal outcomes, in the DHCA group the mean serum creatinine levels were $1.25 \pm 1.03 \mathrm{mg} / \mathrm{dL}$ preoperatively and $1.31 \pm 0.64 \mathrm{mg} / \mathrm{dL}$ postoperatively, with an average change in value of $0.06 \pm 0.60 \mathrm{mg} / \mathrm{dL}$. In the MHCA group, creatinine levels were $1.26 \pm 0.98 \mathrm{mg} / \mathrm{dL}$ preoperatively and $1.43 \pm 0.95 \mathrm{mg} / \mathrm{dL}$ postoperatively, with an average change of $0.15 \pm 0.72 \mathrm{mg} / \mathrm{dL}$. In the DHCA group, 11 patients $(14 \%)$ had preoperative renal dysfunction, 13 patients $(17 \%)$ had postoperative AKI defined by AKIN criteria, 2 patients $(3 \%)$ required temporary hemodialysis, and none required dialysis after discharge. In the MHCA group, 19 patients $(13 \%)$ had preoperative renal dysfunction, 27 patients $(19 \%)$ had postoperative AKI defined by AKIN criteria, 2 patients $(1 \%)$ required temporary hemodialysis, and 1 patient $(1 \%)$ required dialysis after discharge. There were no significant differences in postoperative renal outcomes between the 2 groups.

Multivariate linear regression was performed for outcomes with continuous values. The mean LOS was $16 \pm 13$ days in the DHCA group versus $14 \pm 12$ days in the MHCA group $(P=.39)$. No significant difference was observed between groups in the amount of transfused packed red blood cells, fresh-frozen plasma, platelets (pooled and apheresis), or cryoprecipitate used (Table 3). 
TABLE 2. Postoperative outcomes in patients $(N=221)$ undergoing aortic arch repair with DHCA or MHCA with antegrade cerebral perfusion

\begin{tabular}{|c|c|c|c|c|c|}
\hline Postoperative outcome & DHCA $(n=78)$ & $\operatorname{MHCA}(n=143)$ & $\boldsymbol{P}$ & OR & $\mathbf{9 5} \% \mathbf{C I}$ \\
\hline 30-d mortality, $\%$ & 9 & 2 & .02 & 4.7 & $1.2-18.6$ \\
\hline In-hospital death, $\%$ & 8 & 1 & .005 & 9.3 & $1.1-81.6$ \\
\hline Stroke, $\%$ & 8 & 3 & .09 & 3.4 & 0.9-12.6 \\
\hline Postoperative MI, \% & 1 & 1 & .6 & 0.5 & $0.3-7.6$ \\
\hline Bleeding necessitating reoperation, $\%$ & 3 & 7 & .27 & 0.4 & $0.1-2.0$ \\
\hline Hospital stay, d & $16 \pm 12$ & $14 \pm 12$ & .39 & - & - \\
\hline Postoperative serum creatinine level, $\mathrm{mg} / \mathrm{dL}$ & $1.31 \pm 0.64$ & $1.43 \pm 0.95$ & .30 & - & - \\
\hline Preoperative-postoperative change in serum creatinine, $\mathrm{mg} / \mathrm{dL}$ & $0.06 \pm 0.60$ & $0.15 \pm 0.72$ & .38 & - & - \\
\hline Postoperative AKI by AKIN criteria, \% & 17 & 19 & .89 & 1.0 & $0.4-2.0$ \\
\hline Postoperative AKI by RIFLE criteria, $\%$ & 12 & 12 & .94 & 1.0 & $0.4-2.4$ \\
\hline Postoperative dialysis, $\%$ & 3 & 2 & .84 & 0.8 & $0.1-5.9$ \\
\hline
\end{tabular}

RIFLE, Risk, Injury, Failure, Loss, and End-stage kidney diseases; $D H C A$, deep hypothermic circulatory arrest; $M H C A$, moderate hypothermic circulatory arrest; $O R$, odds ratio; $C I$, confidence interval; $M I$, myocardial infarction; $A K I$, acute kidney injury; $A K I N$, acute kidney injury network.

\section{DISCUSSION}

The ideal HCA temperature for surgical repair of the aortic arch during ACP remains unclear. Although previous animal and clinical studies have suggested that MHCA with ACP may be associated with better neurologic outcomes than DHCA, ${ }^{3,13-16}$ other investigators have not confirmed these findings. ${ }^{17-19}$ Our data suggest that MHCA with ACP is associated with lower hospital and 30-day mortality rates, shorter $\mathrm{CPB}$ time, and fewer neurologic sequelae than DHCA, after adjustment for patient demographics, preoperative risk factors and medications, and intraoperative variables. The 30-day all-cause mortality rate was 4-fold higher in patients who underwent DHCA with ACP instead of MHCA with ACP. The stroke incidence was nearly 3-fold higher in the DHCA group than in the MHCA group ( $8 \%$ vs $3 \%$ ), although this difference was not significant.

Since the introduction of aortic arch replacement in the 1950s by DeBakey and Cooley, ${ }^{20}$ the 2 main methods of brain protection during this procedure have been different degrees of hypothermia and adjuncts for cerebral perfusion. Deep HCA at a body temperature of $18^{\circ} \mathrm{C}$ was first used for successful aortic arch reconstruction in $1975 .{ }^{21}$ Because DHCA dramatically reduces cerebral oxygen requirements, it enabled the development of modern aortic surgery. However, because the safe duration of arrest is limited by the risk of neurologic sequelae, ${ }^{4}$ restricting arch exclusion to 30 to

TABLE 3. Perioperative transfusion requirements $(48 \mathrm{hr})$ in patients $(\mathbf{N}=221)$ undergoing surgical aortic arch repair with DHCA or MHCA with antegrade cerebral perfusion

\begin{tabular}{lccc}
\hline Perioperative transfusion & DHCA $(\mathbf{n}=\mathbf{7 8})$ & MHCA $(\mathbf{n}=\mathbf{1 4 3})$ & $\boldsymbol{P}$ \\
\hline Packed red blood cells, units & $5.6 \pm 5.3$ & $4.6 \pm 4.2$ & .15 \\
Fresh-frozen plasma, units & $5.4 \pm 5.7$ & $4.6 \pm 4.5$ & .24 \\
Pooled platelets, units & $11.5 \pm 12.5$ & $13.3 \pm 10.7$ & .28 \\
Apheresis platelets, doses & $1.2 \pm 1.8$ & $1.1 \pm 1.7$ & .89 \\
Pooled cryoprecipitate, doses & $0.9 \pm 1.3$ & $1.1 \pm 1.1$ & .23 \\
\hline
\end{tabular}

DHCA, Deep hypothermic circulatory arrest; $M H C A$, moderate hypothermic circulatory arrest.
40 minutes has emerged as a guideline for the safe use of an HCA-only strategy. ${ }^{22}$ In the 1990 s, efforts to extend the protected time for aortic arch repair produced the concepts of ACP and retrograde cerebral perfusion, which were used during HCA. ${ }^{12,23}$ Both techniques continue to have their proponents, but retrograde cerebral perfusion seems to have fallen out of favor in the past decade. Multiple studies have shown that ACP extends the safe duration of arch intervention beyond what HCA alone provides, ${ }^{24,25}$ especially when the distal arch repair extends beyond 30 minutes. However, the ideal core temperature for HCA with ACP has yet to be determined.

In an effort to improve patient outcomes after aortic arch replacement, we examined the impact of the temperature used during HCA with ACP on specific perioperative measures. We found that temperature lower than $20^{\circ} \mathrm{C}$ was associated with a 9-fold increase in hospital death, as well as significant increases in 30-day mortality rates and CPB time and a trend toward an increased stroke incidence. Temperature did not, however, have a significant effect on transfusion requirements or postoperative renal function. We chose $20^{\circ} \mathrm{C}$ as the division between moderate and deep hypothermia because a body temperature lower than $20^{\circ} \mathrm{C}$ commonly is defined as profound or deep hypothermia, and because the cooling continued until electrocerebral silence was seen on multichannel electroencephalography. Although temperatures of approximately $23^{\circ} \mathrm{C}$ would characterize severe hypothermia in the general population, such temperatures are more moderate than the temperatures traditionally achieved during HCA.

Although deep hypothermia confers the benefit of minimizing metabolic demand when energy substrates are limited, this level of hypothermia may have many adverse effects. Evidence from both clinical and animal studies suggests that deep hypothermia with rewarming alone is associated with neuronal injury. ${ }^{7}$ Furthermore, Warren et $\mathrm{al}^{7}$ reported that neuronal death from deep hypothermia and rewarming was caused by adenosine triphosphate loss, 
glutamate receptor activation, and $\mathrm{Ca}^{2+}$ influx. Deep hypothermia also may impair microvascular perfusion and disrupt the blood-brain barrier. ${ }^{2,13}$ Larger vessels may be affected by deep hypothermia as well. One group reported that the antegrade cerebral blood flow shunted away from capillaries increases during deep hypothermia. ${ }^{26}$ These effects may explain some of our DHCA patients' adverse outcomes. Our findings also may reflect a more systemic underlying process that is triggered by deep hypothermia on $\mathrm{CPB}$, such as inflammation that leads to organ damage. ${ }^{6}$ Shorter CPB times secondary to a shorter rewarming period in the MHCA group may result in less cellular injury, quicker recovery, and less early mortality risk. Interestingly, Khaladj et $\mathrm{al}^{14}$ found that hypothermia at $20^{\circ} \mathrm{C}$ provides better brain protection than a profound hypothermic temperature of $10^{\circ} \mathrm{C}$ in a pig model of HCA with ACP. Furthermore, it has been reported that mild or moderate cerebral hypothermia induced immediately after cardiac arrest improves cerebral outcome, whereas deep hypothermia can worsen cerebral and cardiac outcomes. ${ }^{27}$ It is unlikely that our results are related directly to differences in surgical technique because the same surgical team performed all of the surgeries. In addition, the lowest temperature was standardized during each era of the study and was not applied selectively to certain (eg, sicker) patients.

Although DHCA prolongs CPB time and may reduce the activity of enzymes involved in platelet activation pathways and clotting factors, both of which can increase transfusion requirements after $\mathrm{DHCA},{ }^{3}$ the transfusion requirement did not differ significantly between the DHCA and MHCA groups. Similarly, there was no observed difference in the incidence of surgical re-exploration. Although these data suggest that choosing DHCA or MHCA does not affect the rate of transfusion or bleeding complications, these data have to be interpreted with caution because of our study's relatively small sample size.

Although ACP provides energy substrates to the brain during HCA, the rest of the body is subjected to total ischemia. Indeed, vascular endothelial dysfunction and apoptosis resulting from $\mathrm{CPB}$ and whole-body systemic ischemia-reperfusion have been implicated in the pathophysiology of multisystem organ failure after DHCA. ${ }^{2}$ Therefore, we evaluated renal function by measuring preoperative and postoperative serum creatinine levels to examine the renal effects of HCA at more moderate temperatures. No significant differences were observed in the risk of postoperative AKI (according to the AKIN and Risk, Injury, Failure, Loss, and End-stage kidney diseases criteria) between the moderate and deep HCA groups. Similarly, with respect to spinal cord injury, no difference in the paraplegia incidence was observed in either group. Together, these data suggest that lower-body end-organs can tolerate brief periods of ischemia when moderate HCA is used for aortic arch surgery.
This study was limited in that it was a retrospective cohort study from a single institution. Most patients who underwent surgery earlier in the study period were exposed to deep hypothermia, whereas the more recent patients underwent moderate hypothermia. Therefore, it is possible that perioperative management techniques changed slightly over time, potentially biasing our results. In contrast, all of the surgeries were performed by a single surgeon with many years of experience in aortic arch surgery; therefore, it is unlikely that any changes over time in surgeon technique or skill affected the results. Also, although all patients with overt clinical evidence of stroke underwent a cerebral imaging study to confirm the stroke, cerebral imaging was not performed in patients without clinical indicators of stroke. Some regions of the brain, such as the pons and the cerebellum, may be more sensitive to ischemic injury, and subtle clinical changes in these areas may not have been detected and recorded. ${ }^{28}$ In addition, despite our careful use of logistic regression models to adjust for potential confounders that may have affected outcomes, other immeasurable factors nonetheless may have biased the results.

In conclusion, this study showed that, in patients who undergo aortic arch surgery, MHCA with ACP is associated with lower hospital and 30-day mortality rates, shorter CPB time, and fewer neurologic sequelae than DHCA. However, MHCA and DHCA are associated with similar transfusion requirements and postoperative renal function.

We thank Stephen N. Palmer, PhD, ELS, senior scientific medical writer at the Texas Heart Institute, for assistance in the preparation of the manuscript.

\section{References}

1. Todd MM, Warner DS. A comfortable hypothesis reevaluated. Cerebral metabolic depression and brain protection during ischemia. Anesthesiology. 1992;76:161-4.

2. Cooper WA, Duarte IG, Thourani VH, Nakamura M, Wang NP, Brown WM III, et al. Hypothermic circulatory arrest causes multisystem vascular endothelial dysfunction and apoptosis. Ann Thorac Surg. 2000;69:696-703.

3. Kamiya H, Hagl C, Kropivnitskaya I, Bothig D, Kallenbach K, Khaladj N, et al. The safety of moderate hypothermic lower body circulatory arrest with selective cerebral perfusion: a propensity score analysis. J Thorac Cardiovasc Surg. 2007; 133:501-9.

4. Svensson LG, Crawford ES, Hess KR, Coselli JS, Raskin S, Shenaq SA, et al. Deep hypothermia with circulatory arrest: determinants of stroke and early mortality in 656 patients. J Thorac Cardiovasc Surg. 1993;106:19-28.

5. DeLeon SY, Thomas C, Roughneen PT, King N, Lehne R, DeLeon AM, et al. Experimental evidence of cerebral injury from profound hypothermia during cardiopulmonary bypass. Pediatr Cardiol. 1998;19:398-403.

6. Qing M, Vazquez-Jimenez JF, Klosterhalfen B, Sigler M, Schumacher K, Duchateau J, et al. Influence of temperature during cardiopulmonary bypass on leukocyte activation, cytokine balance, and post-operative organ damage. Shock. 2001; 15:372-7.

7. Warren DE, Bickler PE, Clark JP, Gregersen M, Brosnan H, McKleroy W, et al. Hypothermia and rewarming injury in hippocampal neurons involve intracellular $\mathrm{Ca}^{2+}$ and glutamate excitotoxicity. Neuroscience. 2012;207:316-25.

8. Fergusson DA, Hebert PC, Mazer CD, Fremes S, MacAdams C, Murkin JM, et al. A comparison of aprotinin and lysine analogues in high-risk cardiac surgery. N Engl J Med. 2008;358:2319-31.

9. Levey AS, Coresh J, Balk E, Kausz AT, Levin A, Steffes MW, et al. National Kidney Foundation practice guidelines for chronic kidney disease: evaluation, classification, and stratification. Ann Intern Med. 2003;139:137-47. 
10. Bellomo R, Ronco C, Kellum JA, Mehta RL, Palevsky P. Acute renal failure definition, outcome measures, animal models, fluid therapy and information technology needs: the Second International Consensus Conference of the Acute Dialysis Quality Initiative (ADQI) Group. Crit Care. 2004;8:R204-12.

11. Mehta RL, Kellum JA, Shah SV, Molitoris BA, Ronco C, Warnock DG, et al. Acute Kidney Injury Network: report of an initiative to improve outcomes in acute kidney injury. Crit Care. 2007;11:R31.

12. LeMaire SA, Price MD, Parenti JL, Johnson ML, Lay AD, Preventza O, et al. Early outcomes after aortic arch replacement by using the Y-graft technique. Ann Thorac Surg. 2011;91:700-7.

13. Allibhai T, DiGeronimo R, Whitin J, Salazar J, Yu TT, Ling XB, et al. Effects of moderate versus deep hypothermic circulatory arrest and selective cerebral perfusion on cerebrospinal fluid proteomic profiles in a piglet model of cardiopulmonary bypass. J Thorac Cardiovasc Surg. 2009;138:1290-6.

14. Khaladj N, Peterss S, Oetjen P, von Wasielewski R, Hauschild G, Karck M, et al. Hypothermic circulatory arrest with moderate, deep or profound hypothermic selective antegrade cerebral perfusion: which temperature provides best brain protection? Eur J Cardiothorac Surg. 2006;30:492-8.

15. Urbanski PP, Lenos A, Bougioukakis P, Neophytou I, Zacher M, Diegeler A. Mild-to-moderate hypothermia in aortic arch surgery using circulatory arrest: a change of paradigm? Eur J Cardiothorac Surg. 2012;41:185-91.

16. Numata S, Tsutsumi Y, Monta O, Yamazaki S, Seo H, Sugita R, et al. Aortic arch repair with antegrade selective cerebral perfusion using mild to moderate hypothermia of more than 28 degrees C. Ann Thorac Surg. 2012;94:90-5; discussion 95-6.

17. Pacini D, Leone A, Di Marco L, Marsilli D, Sobaih F, Turci S, et al. Antegrade selective cerebral perfusion in thoracic aorta surgery: safety of moderate hypothermia. Eur J Cardiothorac Surg. 2007;31:618-22.

18. Salazar J, Coleman R, Griffith S, McNeil J, Young H, Calhoon J, et al. Brain preservation with selective cerebral perfusion for operations requiring circulatory arrest: protection at $25^{\circ} \mathrm{C}$ is similar to $18^{\circ} \mathrm{C}$ with shorter operating times. Eur J Cardiothorac Surg. 2009;36:524-31.
19. Sasaki T, Boni L, Riemer RK, Yeung JT, Ramamoorthy C, Beckman R, et al Cerebral oxygen metabolism during total body flow and antegrade cerebral perfusion at deep and moderate hypothermia. Artif Organs. 2010;34:980-6.

20. DeBakey ME, Crawford ES, Cooley DA, Morris GC Jr. Successful resection of fusiform aneurysm of aortic arch with replacement by homograft. Surg Gynecol Obstet. 1957;105:657-64

21. Griepp RB, Stinson EB, Hollingsworth JF, Buehler D. Prosthetic replacement of the aortic arch. J Thorac Cardiovasc Surg. 1975;70:1051-63.

22. Gega A, Rizzo JA, Johnson MH, Tranquilli M, Farkas EA, Elefteriades JA Straight deep hypothermic arrest: experience in 394 patients supports its effectiveness as a sole means of brain preservation. Ann Thorac Surg. 2007;84:759-66

23. Kazui T, Inoue N, Yamada O, Komatsu S. Selective cerebral perfusion during operation for aneurysms of the aortic arch: a reassessment. Ann Thorac Surg. 1992;53:109-14.

24. Kruger T, Weigang E, Hoffmann I, Blettner M, Aebert H. Cerebral protection during surgery for acute aortic dissection type A: results of the German Registry for Acute Aortic Dissection Type A (GERAADA). Circulation. 2011;124: 434-43.

25. Shihata M, Mittal R, Senthilselvan A, Ross D, Koshal A, Mullen J, et al. Selective antegrade cerebral perfusion during aortic arch surgery confers survival and neuroprotective advantages. J Thorac Cardiovasc Surg. 2011;141:948-52.

26. Usui A, Oohara K, Murakami F, Ooshima H, Kawamura M, Murase M. Body temperature influences regional tissue blood flow during retrograde cerebral perfusion. J Thorac Cardiovasc Surg. 1997;114:440-7.

27. Weinrauch V, Safar P, Tisherman S, Kuboyama K, Radovsky A. Beneficial effect of mild hypothermia and detrimental effect of deep hypothermia after cardiac arrest in dogs. Stroke. 1992;23:1454-62.

28. Strauch JT, Haldenwang PL, Mullem K, Schmalz M, Liakopoulos O, Christ H et al. Temperature dependence of cerebral blood flow for isolated regions of the brain during selective cerebral perfusion in pigs. Ann Thorac Surg. 2009; 88:1506-13 